\title{
Nondissipative Spin Hall Effect via Quantized Edge Transport
}

\author{
L. Sheng, ${ }^{1}$ D. N. Sheng, ${ }^{2}$ C. S. Ting, ${ }^{1}$ and F. D. M. Haldane ${ }^{3}$ \\ ${ }^{1}$ Department of Physics and Texas Center for Superconductivity, University of Houston, Houston, Texas 77204, USA \\ ${ }^{2}$ Department of Physics and Astronomy, California State University, Northridge, California 91330, USA \\ ${ }^{3}$ Department of Physics, Princeton University, Princeton, New Jersey 08544, USA
}

(Received 22 June 2005; published 19 September 2005)

\begin{abstract}
The spin Hall effect in a two-dimensional electron system on honeycomb lattice with both intrinsic and Rashba spin-orbit couplings is studied numerically. Integer quantized spin Hall conductance is obtained at the zero Rashba coupling limit when electron Fermi energy lies in the energy gap created by the intrinsic spin-orbit coupling, in agreement with recent theoretical prediction. While nonzero Rashba coupling destroys electron spin conservation, the spin Hall conductance is found to remain near the quantized value, being insensitive to disorder scattering, until the energy gap collapses with increasing the Rashba coupling. We further show that the charge transport through counterpropagating spin-polarized edge channels is well quantized, which is associated with a topological invariant of the system.
\end{abstract}

DOI: $10.1103 /$ PhysRevLett.95.136602

The proposals of intrinsic spin Hall effect (SHE) in a Luttinger spin-orbit (SO) coupled three-dimensional $p$-doped semiconductor [1] and in a Rashba SO coupled two-dimensional electron system (2DES) [2] have stimulated many subsequent research activities [3-18]. The SHE may potentially provide a purely electrical means to manipulate electron spins without use of ferromagnetic materials or a magnetic field. The SHE in these systems is dissipative because of nonzero longitudinal conductance [9] and exhibits nonuniversal behavior in the presence of disorder [5,6,10-17], which is naturally distinct from the conventional integer quantum Hall effect (IQHE). In particular, it is found $[5,6,16]$ that the bulk SHE in the twodimensional Rashba model may be destroyed by any weak disorder in infinite samples. It is of both fundamental and practical interest to search for nondissipative SHE with universal properties similar to the IQHE, in light of the fact that IQHE can exist in nature in the absence of magnetic field, as first predicted by Haldane [19].

A class of band insulators with SO coupling was suggested as possible candidates for nondissipative SHE [9]. Interestingly, Kane and Mele proposed [20] that the intrinsic SO coupling in single-layer graphene films may give rise to an integer quantized SHE (IQSHE). The intrinsic SO coupling conserves electron spin $s_{z}$. The independent subsystems of two spin directions $\sigma=\uparrow$ and $\downarrow$ are each equivalent to Haldene's spinless IQHE model [19] on honeycomb lattice without magnetic field. They contribute quantized Hall conductances $e^{2} / h$ and $-e^{2} / h$, respectively, when the electron Fermi energy lies inside the energy gap created by the SO coupling. While the charge Hall conductances cancel out, the total spin Hall conductance (SHC) is quantized to $\sigma_{s H}=2$ in units of $e / 4 \pi$. We recall that each subsystem can be classified by an integer Chern number [19], which equals the Hall conductance of the subsystem in units of $e^{2} / h$, and is conserved without spin-mixing interactions. Upon coupling the two subsystems, only the total Chern number (as a well-known topo-
PACS numbers: 72.10. $-\mathrm{d}, 71.70 . \mathrm{Ej}, 72.25 .-\mathrm{b}, 73.43 . \mathrm{Cd}$

logical invariant) is conserved, which is trivially zero as the total Hall conductance vanishes. Therefore, the conservation of electron $s_{z}$ appears to be important to the IQSHE. It is unclear whether the topological SHE could survive if electron $s_{z}$ conservation is destroyed, e.g., by the Rashba SO coupling, which usually exists in a 2DES due to asymmetry in the confining potential. Furthermore, disorder effect in the class of insulating SHE systems has not been studied so far. It is highly desirable to investigate these important issues.

In this Letter, the SHE in the 2D honeycomb lattice model including the intrinsic and Rashba SO couplings is studied numerically. By using the multiprobe LandauerBüttiker formula, we show that the SHC remains nearly quantized in the presence of finite Rashba coupling and disorder scattering until the energy gap collapses. We further show that the charge transport through spinpolarized edge channels is well quantized even for nonzero Rashba coupling, which accounts for the robustness of the SHE. The SHC in samples with close-boundary conditions is also calculated by using the Kubo formula, whereby the SHE is shown to be a stable bulk effect instead of a boundary effect. Our work provides the first numerical demonstration of robust nondissipative SHE in a spin nonconservative 2 DES in the presence of disorder. The nontrivial topological origin of this nondissipative transport regime is also discussed.

The Hamiltonian for a 2DES on a honeycomb lattice can be written as [19-21]

$$
\begin{aligned}
H= & -t \sum_{\langle i j\rangle} c_{i}^{\dagger} c_{j}+\frac{2 i}{\sqrt{3}} V_{\mathrm{SO}} \sum_{\langle i j\rangle\rangle} c_{i}^{\dagger} \boldsymbol{\sigma} \cdot\left(\mathbf{d}_{k j} \times \mathbf{d}_{i k}\right) c_{j} \\
& +i V_{\mathrm{R}} \sum_{\langle i j\rangle} c_{i}^{\dagger} \hat{\mathbf{e}}_{z} \cdot\left(\boldsymbol{\sigma} \times \mathbf{d}_{i j}\right) c_{j}+\sum_{i} \epsilon_{i} c_{i}^{\dagger} c_{i},
\end{aligned}
$$

where $c_{i}^{\dagger}=\left(c_{i \dagger}^{\dagger}, c_{i \downarrow}^{\dagger}\right)$ are electron creation operators, and $\boldsymbol{\sigma}$ are the Pauli matrices. The first term is the usual nearest neighbor hopping term. The second term is the intrinsic SO 
coupling allowed by the symmetries of the honeycomb lattice [19-21] with $i$ and $j$ as two next nearest neighbor sites, where $k$ is the only common nearest neighbor of $i$ and $j$, and $\mathbf{d}_{i k}$ is a vector pointing from $k$ to $i$. The third term is the Rashba SO coupling with $\hat{\mathbf{e}}_{z}$ a unit vector in the $z$ direction. The last term describes nonmagnetic disorder, where $\epsilon_{i}$ is a random on-site potential uniformly distributed in the interval $[-W / 2, W / 2]$. The distance between nearest neighbor sites is taken to be unity. We mention that honeycomb lattice may be realized in other microstructures as well as in single-layer graphene films [22]. For example, in a triangular antidot lattice created at a semiconductor heterointerface by using artificial periodic repulsive potentials, the electrons can be restricted into the region of a honeycomb sublattice [23]. If we switch off the Rashba coupling by setting $V_{\mathrm{R}}=0$, Eq. (1) reduces to a twocomponent Haldane's model [19], which is expected to display $\sigma_{s H}=2$ IQSHE [20].

We consider a four-probe spin Hall setup as illustrated in Fig. 1, where a rectangular honeycomb lattice sample is connected with four ideal semi-infinite leads. The actual system used in our calculations will have the same aspect ratios as Fig. 1 with enlarged sizes. To specify the system size, the sample is divided into $L_{y}$ horizontal chains with $L_{x}$ sites in each chain, as indicated by the dotted lines in Fig. 1. The total number of sites in the sample is denoted as $N=L_{x} \times L_{y}$. For simplicity, the leads are assumed to have a square lattice structure with only nearest neighbor hopping $t$. The spin currents are well defined in the leads, where no SO interactions exist. The SHC is given by twice the ratio of the spin current in lead 3 to the voltage drop $V$ between leads 0 and 1 . Here, a factor 2 is used to properly eliminate the effect of the contact resistances between the leads and the edge channels in the four-probe setup [20]. The linear SHC is calculated exactly by using the multiprobe Landauer-Büttiker formula [12-14,24]

$$
\sigma_{s H}=\frac{e}{4 \pi} \sum_{\sigma \sigma^{\prime}} \sigma\left(T_{30}^{\sigma \sigma^{\prime}}-T_{31}^{\sigma \sigma^{\prime}}\right)
$$

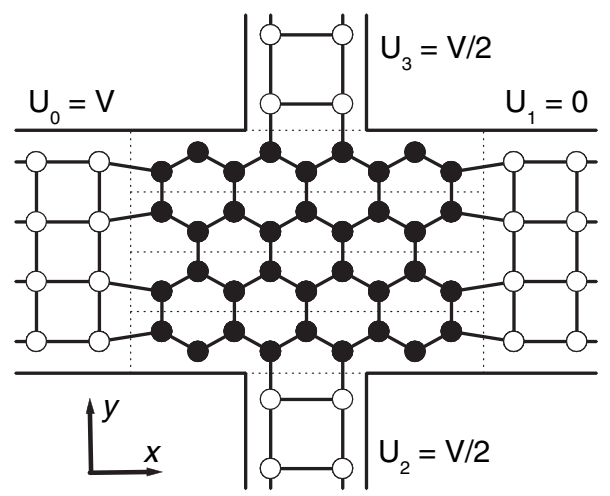

FIG. 1. The four-probe spin Hall bar setup used for calculating the SHC. Solid circles represent the sites in the sample, and open circles stand for the sites in the leads. $U_{l}$ is the electrical voltage in lead $l$. where $T_{l l^{\prime}}^{\sigma \sigma^{\prime}}$ is the spin-resolved electron transmission coefficient from spin $\sigma^{\prime}$ channels in lead $l^{\prime}$ to spin $\sigma$ channels in lead $l$ at the Fermi energy $E$.

In Fig. 2(a), we show the electron density of states (DOS) calculated in the momentum space for a large clean bulk sample. The SHE within the energy gap is our main area of interest. For a sample at half filling, such as an undoped graphene film, the presence of weak disorder can pin the electron Fermi energy inside the gap. We note that the actual electron DOS of the sample in the four-probe setup may be slightly modified from that shown in Fig. 2(a) because of the connection with the leads. In Fig. 2(b), the calculated SHC is shown as a function of electron Fermi energy $E$ for sample size $N=129 \times 64$ and several different strengths of the Rashba coupling $V_{\mathrm{R}}$. At $V_{\mathrm{R}}=0$, the energy gap in the DOS for a clean bulk sample is between $-0.52 t$ to $0.52 t$. As expected, the SHC in Fig. 2(b) is well quantized to integer 2 in the main region of the gap. As $V_{R}$ increases to $0.1 t$, the gap shrinks to $-0.23 t$ to $0.51 t$. The SHC within the gap deviates from the quantized value slightly, showing very small fluctuations with $E$, in contrast to the strong fluctuations outside the gap. The same feature is observed for $V_{\mathrm{R}}=0.2 t$ and $0.3 t$.

The effect of disorder is studied by fixing electron Fermi energy at $E=0.4 t$. For $V_{\mathrm{R}}<0.3 t$ considered below, $E=$ $0.4 t$ is always inside the energy gap of a pure bulk system. The SHC calculated for three different $V_{\mathrm{R}}$ are plotted in Figs. 3(a)-3(c), respectively, as functions of $W$. The results
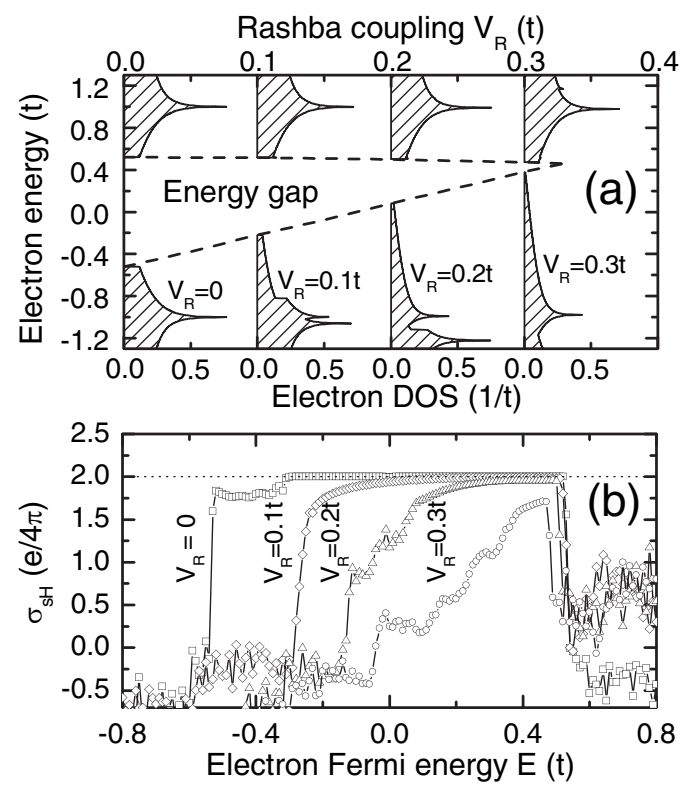

FIG. 2. (a) The curves with solid areas are electron DOS (bottom axis) in a clean bulk sample versus electron energy (left axis) for four different Rashba coupling strengths $V_{\mathrm{R}}$. The dashed lines represent the two edges of the energy gap as functions of the Rashba coupling strength $V_{\mathrm{R}}$ (top axis). (b) Fourprobe $\mathrm{SHC}$ versus electron Fermi energy for some $V_{\mathrm{R}}$ calculated on a $N=129 \times 64$ sample. For both (a) and (b), $W=0$ and $V_{\mathrm{SO}}=0.1 t$. 
for three different sample sizes $(N=65 \times 32,97 \times 48$, and $129 \times 64)$ are shown together for comparison. In Figs. 3(d)-3(f), the corresponding total transmission coefficient $T_{30}=\sum_{\sigma \sigma^{\prime}} T_{30}^{\sigma \sigma^{\prime}}$ is plotted. All $T_{l l^{\prime}}$ for neighboring leads $l$ and $l^{\prime}$ are equal after disorder average by symmetry. They characterize the charge transport between the leads.

Let us first consider the case of zero Rashba coupling $V_{\mathrm{R}}=0$, where the SHC is integer quantized at zero disorder. According to Fig. 3(a), the IQSHE persists for a range of disorder strength $0 \leq W \lesssim 1.2 t$. In the same range, $T_{30}$ in Fig. 3(d) is also quantized to 1 . This is not surprising because at $V_{\mathrm{R}}=0$ the subsystems of the two spin directions are two decoupled IQHE systems. Our result is consistent with electron transport through fully spin-polarized edge channels with spin-dependent chirality. In the IQSHE regime, our calculation yields $T_{30}^{\Uparrow}=$ $T_{13}^{\Uparrow}=T_{21}^{\Uparrow}=T_{02}^{\Uparrow}=1$, representing a left-moving edge mode in the $\sigma=\uparrow$ subsystem, and $T_{03}^{\Downarrow}=T_{31}^{\Downarrow}=T_{12}^{\Downarrow}=$ $T_{20}^{\Downarrow}=1$, corresponding to a right-moving edge mode in the $\sigma=\downarrow$ subsystem. All the other spin-resolved transmission coefficients vanish. Strong disorder $W \gtrsim 1.2 t$ destroys the quantizations of the SHC and transmission coefficients. On the strong disorder side, $T_{30}$ increases rather than decreases with increasing $W$, which signals the collapse of the bulk mobility gap. It is verified but not shown here that, with further increasing $W$, all the transmission coefficients eventually decrease to zero because of electron localization.

Next we look at how the SHC and charge transport evolve with disorder at nonzero Rashba coupling. Remarkably, we see from Figs. 3(e) and 3(f) that $T_{30}$ is still well quantized within a relatively small range of $W$, indicating that the edge modes remain robust. $T_{30}\left(=T_{13}=T_{21}=\right.$

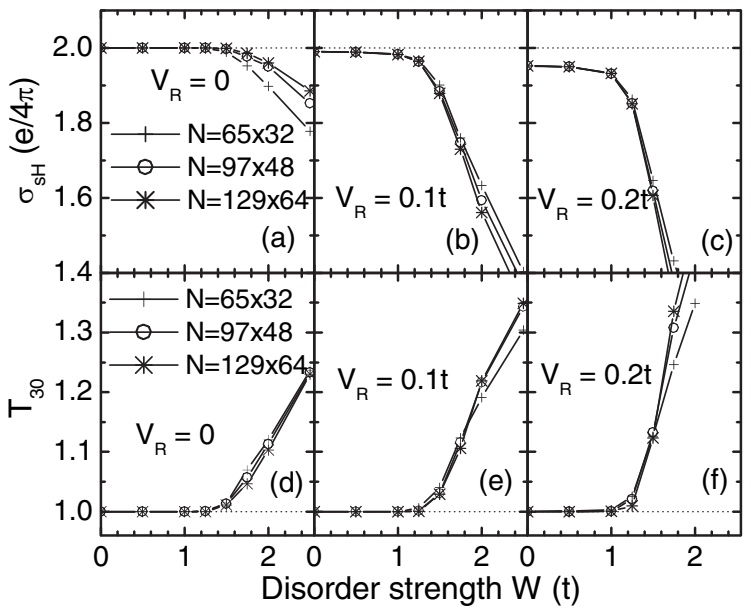

FIG. 3. Four-probe SHC $\sigma_{s H}$ and total transmission coefficient $T_{30}$ for $V_{\mathrm{SO}}=0.1 t, E=0.4 t$, and three different sample sizes. (a) to (c) $\sigma_{s H}$ versus disorder strength $W$ for $V_{\mathrm{R}}=0,0.1 t$, and $0.2 t$, respectively. (d) to (f) corresponding total transmission coefficient $T_{30}$ from lead 0 to lead 3 versus $W$. Here, disorder average is performed over 1000 random configurations.
$\left.T_{02}\right)=1$ relates to the left-moving mode, and $T_{03}\left(=T_{31}=\right.$ $\left.T_{12}=T_{20}\right)=1$ relates to the right-moving mode. However, the spin-resolved transmission coefficients are no longer quantized. For example, at $V_{\mathrm{R}}=0.2 t, W=0.5 t$, and $N=129 \times 64$, we have $T_{30}^{\Uparrow}=0.960, T_{30}^{\Uparrow \downarrow}=0.028$, $T_{30}^{\Downarrow}=0.000$, and $T_{30}^{\Downarrow \uparrow}=0.012$, suggesting that the edge modes become partially spin polarized. Nonetheless, as long as the charge transport is quantized, the SHC stays near the quantized value $\left(\sigma_{s H}=1.95\right.$ for the above parameters), and is robust as it is insensitive to disorder $W$ and independent of sample size $N$, as seen from Figs. 3(b) and 3(c). With further increasing $W, T_{30}$ deviates from the quantized value, and the SHC also decreases rapidly, the system undergoing a transition to a dissipative transport regime.

We have observed that in the presence of not too strong Rashba coupling and disorder, the SHE remains robust and nearly integer quantized. Similar to the IQHE, while the effective current-carrying states are edge states in openboundary systems, the nearly quantized SHE is essentially a stable bulk effect insensitive to boundary conditions or a local Hamiltonian at the boundary. It is of interest to demonstrate this point directly, especially in view of the sensitivity to boundary conditions of the SHE in other metallic models [12-14,17]. We consider a rectangular sample without leads. Periodic boundary conditions are imposed in both the $x$ and $y$ directions. This closeboundary system has translational invariance in the absence of disorder. The Kubo formula [2] is conveniently used to calculate the SHC $\sigma_{s H}$ by exact diagonalization of the system Hamiltonian [16]. Standard spin current operator [2] $J_{y s}^{z}=\left(s_{z} v_{y}+v_{y} s_{z}\right) / 2$ is adopted, where $v_{y}$ is the electron velocity operator in the $y$ direction.

In Fig. 4, the dashed line is the calculated $\sigma_{s H}$ for the ideal case of zero disorder $W=0$ and Rashba coupling $V_{\mathrm{R}}=0$ for system size $N=64 \times 64 . \sigma_{s H}$ is well quantized to 2 in units of $e / 4 \pi$ within the energy gap $(-0.52 t$ to $0.52 t$ ). The lines with symbols are $\sigma_{s H}$ at nonzero $W=t$

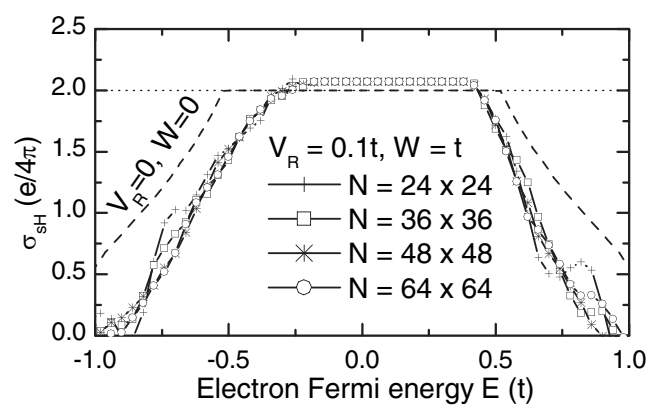

FIG. 4. SHC calculated from the Kubo formula as a function of electron Fermi energy $E$ for $V_{\mathrm{SO}}=0.1 t$. The dashed line is $\sigma_{s H}$ in a clean sample $(W=0)$ with $V_{\mathrm{R}}=0$ and $N=64 \times 64$. The lines with symbols are $\sigma_{s H}$ at $W=t$ and $V_{\mathrm{R}}=0.1 t$ for four different sample sizes. Here, $\sigma_{s H}$ is averaged over 200 disorder configurations for $N=64 \times 64$ and 1000 disorder configurations for the smaller samples. 
and $V_{\mathrm{R}}=0.1 t$ for four different sample sizes from $N=$ $24 \times 24$ to $64 \times 64$. In comparison with the ideal case, the SHC in the gap ( $-0.23 t$ to $0.51 t$ for a pure sample) is not well quantized because of nonzero $V_{\mathrm{R}}$. However, the SHC is very close to the quantized value even in the presence of disorder $W=t$. The four lines collapse in the middle region, an indication that the nearly quantized SHC does not change with increasing sample size, and is thus expected to persist in the thermodynamic limit. We have seen that the SHC in this close-boundary system behaves similarly to that in the four-probe setup, provided that the electron Fermi energy lies inside the energy gap. A nonessential discrepancy is observed that the nearly quantized SHC at $V_{\mathrm{R}}>0$ for the former system is a little greater than $2\left(\sigma_{s H}=2.07\right.$ in Fig. 4), and that for the latter system is smaller than 2. We believe that this discrepancy is caused by the definition of bulk spin current $J_{y s}^{z}$, which is not conservative [6] and hence is not completely equivalent to the spin current measured in leads.

The robust SHE and the quantized charge transfer through edge channels are associated with the nontrivial topological properties of the honeycomb lattice model with SO couplings. In the absence of the Rashba coupling, we have two decoupled subsystems of spin $\sigma=\uparrow$ and $\downarrow$, and each exhibits an integer quantized Hall conductance, $e^{2} / h$ and $-e^{2} / h$. Each subsystem can be classified by an integer Chern number, with $C_{\uparrow}=-C_{\downarrow}=1$. In an open system, there will be two decoupled chiral edge modes moving in opposite directions along the boundary. This picture is substantially altered by the Rashba coupling when the mirror-plane symmetry is destroyed. There are no longer two "types" of electrons due to the spin-mixing effect of the Rashba term. However, while the total Chern number vanishes, the opposite nonzero Chern numbers in the coupled system cannot annihilate each other, as a consequence of "parity anomaly" in the decoupled limit [19]. They coexist and lead to a new topological invariant, which manifests as a pair of edge modes with partial spin polarizations, as indicated by the numerical results. These edge modes are robust in the presence of disorder, until the energy gap collapses, where the lowenergy states merge with their high-energy parity partners [19]. It is worth stressing that the SHC itself is not a topological invariant, which decreases continuously with increasing the strength of the Rashba coupling as the edge states become less spin-polarized. A mathematical description of the new topological invariant will be reported elsewhere.

We thank C.L. Kane and Z. Y. Weng for stimulating discussions. This work is supported by ACS-PRF 41752AC10, Research Corporation Fund CC5643, the NSF
Grant No. DMR-0307170 (D. N. S.), the Robert A. Welch Foundation under Grant No. E-1146 (C.S. T.), and NSF (under MRSEC Grant No. DMR-0213706) at the Princeton Center for Complex Materials (F. D. M. H.).

Note added. - Recently, we found it interesting to notice that in a couple of recent preprints [25], different models for IQSHE are proposed and studied for pure systems. Our Letter represents the first numerical work on the characterization of the SHE in this class of models in the presence of random disorder and coupling between different topological subsystems.

[1] S. Murakami, N. Nagaosa, and S. C. Zhang, Science 301, 1348 (2003); Phys. Rev. B 69, 235206 (2004).

[2] J. Sinova et al., Phys. Rev. Lett. 92, 126603 (2004).

[3] D. Culcer et al., Phys. Rev. Lett. 93, 046602 (2004).

[4] Y. K. Kato et al., Science 306, 1910 (2004); J. Wunderlich et al., Phys. Rev. Lett. 94, 047204 (2005).

[5] J. I. Inoue, G. E. W. Bauer, and L. W. Molenkamp, Phys. Rev. B 70, 041303(R) (2004).

[6] E. G. Mishchenko, A. V. Shytov, and B. I. Halperin, Phys. Rev. Lett. 93, 226602 (2004).

[7] E. I. Rashba, Phys. Rev. B 70, 161201 (2004); 70, 201309(R) (2004).

[8] S.-Q. Shen, Phys. Rev. B 70, 081311(R) (2004).

[9] S. Murakami, N. Nagaosa, and S.C. Zhang, Phys. Rev. Lett. 93, 156804(R) (2004).

[10] S. Murakami, Phys. Rev. B 69, 241202(R) (2004).

[11] K. Nomura et al., Phys. Rev. B 71, 041304(R) (2005).

[12] L. Sheng, D. N. Sheng, and C. S. Ting, Phys. Rev. Lett. 94, 016602 (2005).

[13] B. K. Nikolić, L. P. Zârbo, and S. Souma, cond-mat/ 0408693.

[14] E. M. Hankiewicz, L. W. Molenkamp, T. Jungwirth, and J. Sinova, Phys. Rev. B 70, 241301(R) (2004).

[15] W. Q. Chen, Z. Y. Weng, and D. N. Sheng, cond-mat/ 0502570 .

[16] D. N. Sheng, L Sheng, Z. Y. Weng, and F. D. M. Haldane, cond-mat/0504218.

[17] M. W. Wu and J. Zhou, cond-mat/0503616.

[18] J. P. Hu, B. A. Bernevig, and C. J. Wu, Int. J. Mod. Phys. B 17, 5991 (2003); J. Hu, cond-mat/0502005.

[19] F. D. M. Haldane, Phys. Rev. Lett. 61, 2015 (1988).

[20] C. L. Kane and E. J. Mele, cond-mat/0411737.

[21] M. Onoda and N. Nagaosa, Phys. Rev. Lett. 90, 206601 (2003).

[22] K. S. Novoselov et al., Science 306, 666 (2004).

[23] H. Suzuura and T. Ando, Phys. Rev. Lett. 89, 266603 (2002).

[24] M. Büttiker, Phys. Rev. Lett. 57, 1761 (1986).

[25] B. A. Bernevig and S. C. Zhang, cond-mat/0504147; X.-L. Qi, Y.-S. Wu, and S.C. Zhang, cond-mat/0505308. 